\title{
An algorithm for calculating atomic $D$ states with explicitly correlated Gaussian functions
}

\author{
Keeper L. Sharkey, ${ }^{1}$ Sergiy Bubin, ${ }^{2}$ and Ludwik Adamowicz ${ }^{1,3}$ \\ ${ }^{1}$ Department of Chemistry and Biochemistry, University of Arizona, Tucson, Arizona 85721, USA \\ ${ }^{2}$ Department of Physics and Astronomy, Vanderbilt University, Nashville, Tennessee 37235, USA \\ ${ }^{3}$ Department of Physics, University of Arizona, Tucson, Arizona 85721, USA
}

(Received 21 September 2010; accepted 10 November 2010; published online 27 January 2011)

\begin{abstract}
An algorithm for the variational calculation of atomic $D$ states employing $n$-electron explicitly correlated Gaussians is developed and implemented. The algorithm includes formulas for the first derivatives of the Hamiltonian and overlap matrix elements determined with respect to the Gaussian nonlinear exponential parameters. The derivatives are used to form the energy gradient which is employed in the variational energy minimization. The algorithm is tested in the calculations of the two lowest $D$ states of the lithium and beryllium atoms. For the lowest $D$ state of $\mathrm{Li}$ the present result is lower than the best previously reported result. () 2011 American Institute of Physics. [doi:10.1063/1.3523348]
\end{abstract}

\section{INTRODUCTION}

Very accurate quantum mechanical calculations of the ground and excited states of small atoms have always provided the testing ground for new computational methods for atomic calculations. The testing has been possible due to the availability of very accurate gas-phase spectra of these systems. An important group of states for which such high accuracy experimental data are available are $D$ states. For example, the NIST atomic spectra database ${ }^{1}$ among the 182 states of the lithium atom lists ten ${ }^{2} D$ states which correspond to the electron configurations $1 s^{2} n d$, where $n=3,4, \ldots, 12$. For the beryllium atom among the 219 levels listed there are $11{ }^{1} D$ states and $10^{3} D$ states. The lowest ${ }^{1} D$ state corresponds to the electron configuration $1 s^{2} 2 p^{2}$ and the rest to the configurations $1 s^{2} n d^{2}, n=3,4, \ldots, 12$. The ${ }^{3} D$ states correspond to the configurations $1 s^{2} n d^{2}, n=3,4, \ldots, 12$.

A literature search reveals that only the lowest ${ }^{2} D$ state of lithium has been calculated with high accuracy. The best to date variational energy for this state was reported by Yan and Drake $^{2}$ and it is equal to -7.335 523541 10(43) a.u. (an extrapolated result). There are no calculations with similar accuracy for the $D$ states of beryllium. Part of the reason for the lack of calculations of these states is due to the basis set, which for most works concerning atomic levels has involved Hylleraas type functions. These functions, while very effective in the calculations of two- and three-electron atoms, ${ }^{2-7}$ have not yet been extended to four-electron atoms due to difficulties with calculating the Hamiltonian matrix elements.

Another type of basis function that has been very popular in high-accuracy atomic calculations are correlated Gaussian functions that explicitly depend on the interelectron distances. The most accurate results for four- and five-electron atoms have been obtained with those functions. ${ }^{8-11}$ The main advantage of using Gaussians in atomic calculations is due to the simplicity of the Hamiltonian and overlap integrals with those functions, which can be evaluated analytically for an arbitrary number of electrons. However, these functions cannot satisfy the Kato cusps conditions and are too fast decaying at large distances. As the calculations have shown ${ }^{8-11}$ these deficiencies can be effectively remedied by using longer expansions and by performing extensive optimization of the Gaussian nonlinear parameters using the variational approach.

In this work we have derived and implemented algorithms for calculating the Hamiltonian matrix elements with explicitly correlated Gaussian functions for describing $D$ states of small atomic systems. We also derived and implemented algorithms for calculating first derivatives of the matrix elements determined with respect to the Gaussian exponents. These derivatives are used to calculate the energy gradient, which is employed in the variational optimization of the Gaussian parameters. The variational energy minimization is greatly accelerated if the energy gradient is available. To test the algorithms, we have performed calculations of two lowest ${ }^{2} D$ states of lithium and two lowest ${ }^{1} D$ states of beryllium.

In the approach we use in this work we explicitly account for the finite mass of the nucleus in the variational nonrelativistic calculations. This is done by means of using a Hamiltonian that explicitly depends on the masses of all particles including the mass of the nucleus (see Sec. I A). In this way the results change when a different isotope is considered. The approach also allows us to obtain results corresponding to an infinite mass of the nucleus. Such results generated here for lithium in the present work allow for a direct comparison with the calculations performed by Yan and Drake. ${ }^{2}$

\section{A. The Hamiltonian}

We consider an atom with $N$ particles (i.e., $N-1$ electrons and a nucleus). We start with the laboratory-frame nonrelativistic Hamiltonian and we separate out the centerof-mass motion. This is done by introducing an internal Cartesian coordinate system centered at the nucleus. The separation of the center-of-mass motion is rigorous and results in the laboratory Hamiltonian becoming a sum of the operator 
representing the kinetic energy of the center-of-mass motion and the following "internal" Hamiltonian:

$$
\begin{aligned}
\hat{H}= & -\frac{1}{2}\left(\sum_{i=1}^{n} \frac{1}{\mu_{i}} \nabla_{\mathbf{r}_{i}}^{2}+\frac{1}{m_{0}} \sum_{\substack{i, j=1 \\
i \neq j}}^{n} \nabla_{\mathbf{r}_{i}} \cdot \nabla_{\mathbf{r}_{j}}\right) \\
& +\sum_{i=1}^{n} \frac{q_{0} q_{i}}{r_{i}}+\sum_{i>j=1}^{n} \frac{q_{i} q_{j}}{r_{i j}},
\end{aligned}
$$

where $n=N-1, \mathbf{r}_{i}$ is the distance between the $i$ th electron and the nucleus, $m_{0}$ is the nucleus mass and $q_{0}$ is its charge, $q_{i}$ are electron charges, and $\mu_{i}=m_{0} m_{i} /\left(m_{0}+m_{i}\right)$ are electron reduced masses. The Hamiltonian (1) describes the motion of $n$ (pseudo)electrons, whose masses have been changed to the reduced masses, in the central field of the charge of the nucleus. This motion is coupled through the Coulombic interactions,

$$
\sum_{i=1}^{n} \frac{q_{0} q_{i}}{r_{i}}+\sum_{i>j=1}^{n} \frac{q_{i} q_{j}}{r_{i j}}
$$

where $r_{i j}=\left|\mathbf{r}_{j}-\mathbf{r}_{i}\right|$, and through the mass polarization term,

$$
-\frac{1}{2} \sum_{\substack{i, j=1 \\ i \neq j}}^{n}\left(1 / m_{0}\right) \nabla_{\mathbf{r}_{i}} \cdot \nabla_{\mathbf{r}_{j}} .
$$

\section{THE BASIS SET}

In this work we consider atomic $D$ states corresponding to electronic configurations where one or two electrons of the $n$ electron atom is occupying a non- $s$ state. Examples of such states include the above-mentioned ${ }^{2} D 1 s^{2} n d^{1}$ states of lithium and the ${ }^{1} D$ and ${ }^{3} D 1 s^{2} 2 s^{1} n d^{1}$ states of beryllium. To describe an atomic state with a single $d$ electron one, in principle, needs to use the following explicitly correlated Gaussian function:

$$
\phi_{k}=\left(x_{i_{k}}^{2}+y_{i_{k}}^{2}-2 z_{i_{k}}^{2}\right) \exp \left[-\mathbf{r}^{\prime}\left(A_{k} \otimes I_{3}\right) \mathbf{r}\right],
$$

where $i_{k}$ is an electron label whose value can vary from 1 to $n$ and is unique for each basis function. The prime indicates the matrix/vector transpose; this notation is used throughout this work. $A_{k}$ in Eq. (2) is an $n \times n$ symmetric matrix, $\otimes$ is the Kronecker product, $I_{3}$ is a $3 \times 3$ identity matrix, and $\mathbf{r}$ is a $3 n$ vector that has the form

$$
\mathbf{r}=\left(\begin{array}{c}
\mathbf{r}_{1} \\
\mathbf{r}_{2} \\
\vdots \\
\mathbf{r}_{n}
\end{array}\right)=\left(\begin{array}{c}
x_{1} \\
y_{1} \\
z_{1} \\
\vdots \\
x_{n} \\
y_{n} \\
z_{n}
\end{array}\right) .
$$

We will denote $\left(A_{k} \otimes I_{3}\right)$ in Eq. (2) as $\mathbf{A}_{k}$. However, in the wave functions of some of the $D$ states there can be components corresponding to two electrons being in $p$ states coupling to a $D$ state. For example, the lowest ${ }^{1} D$ state of beryllium is dominated by the $1 s^{2} 2 p^{2}$ configuration. Also, the lowest ${ }^{2} D$ state of lithium has a non-negligible contribution from a similar configuration $\left(1 s^{1} 2 p^{2}\right){ }^{2}$ Thus, the Gaussian basis set that can best capture all the angular coupling effects in $D$ states is the following basis:

$\phi_{k}=\left(x_{i_{k}} x_{j_{k}}+y_{j_{k}} y_{i_{k}}-2 z_{i_{k}} z_{j_{k}}\right) \exp \left[-\mathbf{r}^{\prime}\left(A_{k} \otimes I_{3}\right) \mathbf{r}\right]$,

where electron indices $i_{k}$ and $j_{k}$ are either equal or not equal to each other.

We use a general quadratic form $\mathbf{r}^{\prime} \mathbf{W}_{k} \mathbf{r}$ in place of $\left(x_{i_{k}} x_{j_{k}}+y_{i_{k}} y_{j_{k}}-2 z_{i_{k}} z_{j_{k}}\right)$ allowing for a more generalized approach in deriving the matrix elements. With that, our basis functions are

$$
\phi_{k}=\left(\mathbf{r}^{\prime} \mathbf{W}_{k} \mathbf{r}\right) \exp \left[-\mathbf{r}^{\prime} \mathbf{A}_{k} \mathbf{r}\right],
$$

where $\mathbf{W}_{k}$ is a sparse $3 n \times 3 n$ symmetric matrix that for $i_{k}=j_{k}$ comprises only three nonzero elements: $W_{3\left(i_{k}-1\right)+1,3\left(i_{k}-1\right)+1}=1, \quad W_{3\left(i_{k}-1\right)+2,3\left(i_{k}-1\right)+2}=1, \quad$ and $W_{3\left(i_{k}-1\right)+3,3\left(i_{k}-1\right)+3}=-2$, and for $i_{k} \neq j_{k}$ it comprises six elements: $W_{3\left(i_{k}-1\right)+1,3\left(j_{k}-1\right)+1}=W_{3\left(j_{k}-1\right)+1,3\left(i_{k}-1\right)+1}=\frac{1}{2}$, $W_{3\left(i_{k}-1\right)+2,3\left(j_{k}-1\right)+2}=W_{3\left(j_{k}-1\right)+2,3\left(i_{k}-1\right)+2}=\frac{1}{2}, \quad$ and $W_{3\left(i_{k}-1\right)+3,3\left(j_{k}-1\right)+3}=W_{3\left(j_{k}-1\right)+3,3\left(i_{k}-1\right)+3}=1$. It should be noted that, in general, we could have used a nonsymmetric matrix (for $i_{k} \neq j_{k}$ ) $\mathbf{W}_{k}$ with only three nonzero elements (yielding the same quadratic form) since there are only three terms in Eq. (4). However, in practice it is much more convenient to deal with symmetric $\mathbf{W}_{k}$ matrices as the derivations of matrix elements becomes considerably simpler in this case.

As the basis functions used in describing bound states must be square integrable, restrictions must be imposed on the $A_{k}$ matrices. Each $A_{k}$ matrix must be positive definite. Rather than restricting the $A_{k}$ matrix elements, which usually leads to cumbersome constraints, we use the following Cholesky factored form of $A_{k}: A_{k}=L_{k} L_{k}^{\prime}$, where $L_{k}$ is a lower triangular matrix. With this representation, $A_{k}$ is automatically positive definite for any values of $L_{k}$ ranging from $\infty$ to $-\infty$. Thus, the variational energy minimization with respect to the $L_{k}$ parameters can be carried out without any restrictions. It should be noted that the $L_{k} L_{k}^{\prime}$ representation of $A_{k}$ matrix does not limit the flexibility of basis functions, because any symmetric positive matrix can be represented in a Cholesky factored form.

The linear expansion coefficients of the wave function in terms of the basis functions and the elements of the $L_{k}$ matrices are optimization variables. Also, for each basis function the $i_{k}$ and $j_{k}$ indices are optimized. This optimization is only done once for each basis function when the function is first added to the basis set.

\section{THE HAMILTONIAN INTEGRALS AND THE GRADIENT}

In order to fully exploit the sparsity of the $\mathbf{W}_{k}$ matrices and make the calculation more efficient, three cases have been distinguished in the calculation of the Hamiltonian and overlap integrals. The first case concerns the integrals between the basis functions with $i_{k} \neq j_{k}$, the second case the integrals for functions with $i_{k}=j_{k}$, and the third case the mixed integrals. 
Similar separation is applied in the calculation of the energy gradient. As the integrals and the gradient are similar to those published before for atomic states with two $p$ electrons, ${ }^{12,13}$ we only present the final formulas in the Appendix.

As the total atomic wave function must be antisymmetric with respect to the permutation of the electron labels an appropriate symmetry projection needs to be applied to each basis function. In this work we use the spin-free formalism. The Young projection operator, $\hat{Y}$, which imposes proper symmetry, has to be applied only to the spatial part of the wave function and thus to each basis function, $\hat{Y} \phi_{k} . \hat{Y}$ is a linear combination of permutational operators, $\hat{P}_{\gamma}$, and, as the Hamiltonian is invariant with respect to all permutations of the electrons, in the calculation of the overlap and Hamiltonian matrix elements it is easy to arrange that the permutational operators are applied to the ket only. In brief, the ket functions in those matrix elements are operated on with the permutation operator $\hat{P}=\hat{Y}^{\dagger} \hat{Y}$ (the dagger stands for conjugate), where the $\hat{Y}$ operator can be derived using the appropriate Young tableaux for the state under consideration. For lithium in a ${ }^{2} D$ and beryllium in a ${ }^{1} D$ state the Young operators can be chosen as $\hat{Y}=\left(\hat{1}+\hat{P}_{34}\right)\left(\hat{1}-\hat{P}_{23}\right)$ and $\hat{Y}=\left(\hat{1}-\hat{P}_{45}\right)\left(\hat{1}-\hat{P}_{23}\right)\left(\hat{1}+\hat{P}_{24}\right)\left(\hat{1}+\hat{P}_{35}\right)$, respectively, where the nucleus is labeled as 1 , and the electrons are labeled as $2,3,4,5, \hat{1}$ is the identity operator, and $\hat{P}_{i j}$ is the permutation of the the $i$ th and $j$ th electron.

\section{A. Total energy and energy gradient}

The optimization of the linear expansion coefficients, the $L_{k}$ Gaussian parameters, and the $i_{k}$ and $j_{k}$ indices, is performed in this work through the minimization of the standard Rayleigh-Ritz variational energy functional. In the minimization the energy gradient is employed. The calculation for each state is performed independently from other states and the parameters of the basis functions are optimized specifically for that particular state. In the process of generating the basis set, we start with a small randomly selected set of functions and this set is then grown by incremental additions of small groups of functions. The added functions are generated randomly based on the actual distribution of nonlinear parameters of the functions already present in the basis set. At this step a significant number of random candidates are generated and only those that give the best improvement of the total energy are selected. When a new group of functions is added to the set their nonlinear parameters are optimized with the gradientbased method. After this is finished the nonlinear parameters of the entire basis set (one function at a time) are reoptimized.

\section{NUMERICAL TESTS}

The first test of the approach developed in this work concerns the lowest ${ }^{2} D$ state of lithium. As mentioned this state was calculated before by Yan and Drake. ${ }^{2}$ As their calculation was performed with an infinite mass of the lithium nucleus, we also first carried a calculation setting the mass of the nucleus $\left(m_{0}\right)$ to infinity. The convergence of the total energy with the number of Gaussian functions in the ba-
TABLE I. The convergence of the total variational energy of the lowest ${ }^{2} D$ state of lithium with the number of Gaussians obtained in the present infinitenuclear mass variational calculations. The energies are in hartrees.

\begin{tabular}{lc}
\hline \hline Basis set size & Energy \\
\hline 1900 & -7.33552353070 \\
2300 & -7.33552353822 \\
2700 & -7.33552354089 \\
3100 & -7.33552354196 \\
3500 & -7.33552354251 \\
3900 & -7.33552354282 \\
$1673^{\mathrm{a}}$ & -7.33552354035 \\
$\infty^{\mathrm{a}, \mathrm{b}}$ & $-7.33552354110(43)$ \\
\hline \hline
\end{tabular}

${ }^{\mathrm{a}}$ From Ref. 2.

${ }^{\mathrm{b}}$ The energy obtained by extrapolating to infinity in terms of the parameter $\Omega$ used in the integral formulas by Yan and Drake (Ref. 2).

sis set is shown in Table I. As one notices, the energy is converged to about ten significant figures. The energy value obtained in the present calculations with 3900 Gaussians of $-7.33552354282(60)$ a.u. is lower than the previous best variational upper bound of Yan and Drake ${ }^{2}$ by about $2 \times 10^{-9}$ hartree. The Yan and Drake energy is obtained in our calculations with about 2600 Gaussians in the basis set. The lithium test clearly shows that Gaussians can effectively describe the $D$ states of small atomic systems. Also, the agreement between Yan and Drake's and our results give us confidence that the algorithm and its implementation works correctly.

The next test concerns the energy differences between the energies of the two lowest ${ }^{2} \mathrm{D}$ states of ${ }^{7} \mathrm{Li}$ corresponding to the electron configurations $1 s^{2} 3 d^{1}$ and $1 s^{2} 4 d^{1}$ and the ${ }^{7} \mathrm{Li} 1 s^{2} 2 s^{1}$ ground state energy. These energy differences can be compared with the experimental values taken from the NIST atomic spectra database. ${ }^{1}$ Here we used a finite nuclear mass approach, which is possible in our scheme. The convergence of the total nonrelativistic energies with the number of basis functions obtained in those calculations are shown in Table II. We also show in Table II the energy of the ${ }^{7} \mathrm{Li}$ ground state obtained in our previous work ${ }^{14}$ and the energy of ${ }^{7} \mathrm{Li}^{+}$ calculated in this work. The latter value is used to determine the ${ }^{7} \mathrm{Li}$ nonrelativistic ionization potential (IP). The reason we need the ${ }^{7} \mathrm{Li}$ IP is to compare it with the relative energies of the ${ }^{2} D$ states determined with respect to the energy of the ground state. Such a comparison must show that in the limit of exciting the $d$ electron in ${ }^{7} \mathrm{Li}$ to increasingly higher ${ }^{2} \mathrm{D}$ states, the relative energy should converge to the ${ }^{7} \mathrm{Li}$ IP as the electron becomes removed from the atom. Naturally, as only two ${ }^{2} D$ states are calculated in this work and not a wider range of the Rydberg $1 s^{2} n d^{1}$ states is considered, the consistency of the results and not the convergence will be tested.

It should also be mentioned that, as the present results do not include relativistic corrections, the agreement with the experimental values is not expected to be perfect. This is why we need the IP value. If the algorithm implemented in this work is correct, the difference between the experimental and calculated nonrelativistic IPs should be similar to the differences between the calculated relative energies of the two ${ }^{2} D$ states of ${ }^{7} \mathrm{Li}$ and their corresponding experimental counterparts. The 
TABLE II. The convergence of the total variational finite mass energies of the two lowest ${ }^{2} \mathrm{D}$ states of ${ }^{7} \mathrm{Li}$ with the number of Gaussians. The total energies are in hartrees and the ionization potentials (IP) in $\mathrm{cm}^{-1}$.

\begin{tabular}{lccc}
\hline \hline No. of basis functions & ${ }^{7} \mathrm{Li}_{1} s^{2} 3 d^{1}$ & ${ }^{7} \mathrm{Li} 1 s^{2} 4 d^{1}$ & ${ }^{7} \mathrm{Li}^{+} 1 s^{2}$ \\
\hline 2600 & -7.33492730445 & -7.31059523610 & \\
2800 & -7.33492730488 & -7.31059523762 & \\
3000 & -7.33492730514 & -7.31059523865 & \\
3200 & -7.33492730541 & -7.31059523966 & \\
3400 & -7.33492730559 & -7.31059524028 & \\
3600 & -7.33492730575 & -7.31059524076 & \\
$10000^{\mathrm{a}}$ & & & -7.27932151683 \\
$300^{\mathrm{b}}$ & & & -7.27932151940 \\
400 & & & -7.27932151972 \\
500 & 31280.54 & 36620.81 & 43484.60 \\
Energy difference $^{\mathrm{c}}$ & 31283.08 & 36623.38 & 43487.15 \\
Experiment $^{\mathrm{d}}$ & -2.54 & -2.57 & -2.55 \\
$\Delta^{\mathrm{e}}$ & & & \\
\hline \hline
\end{tabular}

${ }^{\mathrm{a}}$ From Ref. 14.

${ }^{\mathrm{b}}$ Calculations for ${ }^{7} \mathrm{Li}^{+}$have been performed in this work.

${ }^{\mathrm{c}}$ Calculated relative to the ground $1 s^{2}{ }^{2} s^{1}$ state of ${ }^{7} \mathrm{Li}$. For the ${ }^{7} \mathrm{Li} 2 \mathrm{D}$ states the energies obtained with $3600 \mathrm{Gaussians}$ were used. For ${ }^{7} \mathrm{Li}{ }^{+}$the energy obtained with $500 \mathrm{Gaussians}$ was used.

${ }^{\mathrm{d}}$ From Ref. 1.

${ }^{\mathrm{e}}$ Determined as the calculated energy difference minus the experimental energy difference.

results presented in Table II show that they are indeed very similar. The IP of ${ }^{7} \mathrm{Li}$ calculated at the nonrelativistic level of $43484.60 \mathrm{~cm}^{-1}$ is lower than the experimental value by $2.55 \mathrm{~cm}^{-1}$. This is very close to the differences between the experimental and calculated relative energies of the two ${ }^{2} D$ states, which are 2.54 and $2.57 \mathrm{~cm}^{-1}$, respectively. Clearly, the relativistic contribution to the relative energies of the ${ }^{2} D$ states of ${ }^{7} \mathrm{Li}$ from the Rydberg $d$ electron is very small. It should be said that the present finite-mass calculations of the two ${ }^{2} \mathrm{D}$ states of ${ }^{7} \mathrm{Li}$ are the first ever performed. Also, these are the first calculations that described the $1 s^{2} 4 d^{12} D^{7} \mathrm{Li}$ state with a similar accuracy as the lower $1 s^{2} 3 d^{12} D$ state.

The last test concerns the two lowest ${ }^{1} D$ states of the beryllium atom $\left({ }^{9} \mathrm{Be}\right)$. This test is performed to show the capability of the method developed in this work to describe
$D$ states of atoms with more than three electrons-the task which is still out of reach for other types of explicitly correlated basis functions. The results of the beryllium calculations are shown in Table III. Here we also show the convergence of the total nonrelativistic energies for the ${ }^{9} \mathrm{Be}$ $1 s^{2} 2 p^{2}$ and $1 s^{2} 2 s^{1} 3 d^{1}{ }^{1} D$ states with the number of basis functions and the relative energies with respect to the ground $1 s^{2} 2 s^{2}{ }^{1} S$ state. As in Table II, the results also include the IP value, as well as the experimental values taken from the NIST atomic spectra database. ${ }^{1}$

First, as one notices, the total variational energies for beryllium ${ }^{1} D$ states are not as well converged with the number of basis functions as the energies for the two ${ }^{2} D$ states of lithium. The lowest energy values corresponding to configurations $1 s^{2} 2 p^{2}$ and $1 s^{2} 2 s^{1} 3 d^{1}$, which we obtained in

TABLE III. The convergence of the total variational finite mass energies of the two lowest ${ }^{1} D$ states of ${ }^{9} \mathrm{Be}$ with the number of Gaussians. The total energies are in hartrees and the energy differences with respect to the ground state in $\mathrm{cm}^{-1}$.

\begin{tabular}{lccc}
\hline \hline No. of basis functions & ${ }^{9} \mathrm{Be} 1 s^{2} 2 p^{2}$ & ${ }^{9} \mathrm{Be} 1 s^{2} 2 s^{1} 3 d^{1}$ & ${ }^{9} \mathrm{Be}^{+} 1 s^{2} 2 s^{1}$ \\
\hline 1200 & -14.4073414805 & -14.3729165342 & \\
1300 & -14.4073436284 & -14.3729182828 & \\
1400 & -14.4073452331 & -14.3729190111 & \\
1500 & -14.4073463769 & -14.3729205728 & \\
1600 & -14.4073479461 & -14.3729213166 & \\
1700 & -14.4073478753 & -14.3729215856 & \\
1800 & -14.4073484882 & -14.3729222593 & -14.3238634944 \\
$10000^{\mathrm{a}}$ & & & 75185.87 \\
$8000^{\mathrm{b}}$ & & & 75192.64 \\
Energy difference $^{\mathrm{c}}$ & 56863.03 & 64418.71 & -6.77 \\
Experiment $^{\mathrm{d}}$ & 56882.43 & 64428.31 & -14.666435504 \\
$\Delta^{\mathrm{e}}$ & -19.40 & -9.60 & \\
\hline \hline
\end{tabular}

${ }^{\mathrm{a}}$ From Ref. 15.

${ }^{\mathrm{b}}$ From Ref. 16.

${ }^{\mathrm{c}}$ Calculated relative to the ground $1 \mathrm{~s}^{2} 2 \mathrm{~s}^{2}$ state of ${ }^{9} \mathrm{Be}$. For the ${ }^{1} D$ Be states the energies obtained with 1800 Gaussians were used

${ }^{\mathrm{d}}$ From Ref. 1.

${ }^{\mathrm{e}}$ Determined as the calculated energy difference minus the experimental energy difference. 
our calculations are-14.407 3484882 and -14.372 9222593 a.u., respectively. We estimate that the remaining uncertainty for these values is of the order of $10^{-6}$ a.u. It would take several thousand Gaussians to converge them to the level of accuracy reached for lithium and such calculations will be carried out in the future. However, even with the present results, the agreement with the experiment for the relative energies is very good. Also the difference between the calculated relative energies and the experimental energies seems to converge to the IP calculated based on very accurate total energies of ${ }^{9} \mathrm{Be}$ and ${ }^{9} \mathrm{Be}^{+}$taken from our previous works. ${ }^{15,16}$ The results show that, unlike for lithium, the relativistic correction to IP of ${ }^{9} \mathrm{Be}$ is almost three times smaller than for the relative energy of the lowest ${ }^{1} D$ state of this atom.

\section{SUMMARY}

The following has been accomplished in this work.

1. An algorithm for nonrelativistic variational calculations of atomic $D$ states has been developed and implemented.

2. The approach has been used to obtain new improved upper bound to the variational infinite-mass energy of the lowest ${ }^{2} D$ state of lithium.

3. High-accuracy lithium calculations have also been performed for the two lowest ${ }^{2} \mathrm{D}$ states of the ${ }^{7} \mathrm{Li}$ isotope using the finite-mass regime enabled by the approach. The results show very good agreement with the experiment.

4. It has been demonstrated that the approach can be used to carry out calculations of $D$ states of an atomic system with more than three electrons.

The work presented here will be extended in the future to study wider range of Rydberg $D$ states of the leading isotopes of lithium and beryllium. The approach will also be used in calculations of $D$ states of atoms and atomic ions with more than four electrons.

\section{APPENDIX A: MATRIX ELEMENTS AND THE GRADIENT}

Below, we show the expressions for the Hamiltonian and overalp matrix elements and the corresponding derivatives with respect to the $L_{k}$ matrix elements. We do not show how those quantities have been derived because the procedure was very similar to that presented in our previous works. $^{12,13}$

Let us first define a quantity common to the overlap, kinetic, and potential energy matrix elements:

$$
\eta=\operatorname{tr}\left[\mathbf{A}_{k l}^{-1} \mathbf{W}_{k} \mathbf{A}_{k l}^{-1} \mathbf{W}_{l}\right],
$$

and a quantity common only to the kinetic energy matrix element:

$$
\tau=\operatorname{tr}\left[A_{k l}^{-1} A_{k} M A_{l}\right] .
$$

In Eq. (A2) $M$ is the mass matrix whose diagonal elements are set to $1 /\left(2 m_{1}\right), 1 /\left(2 m_{2}\right), \ldots, 1 /\left(2 m_{n}\right)$, while the off-diagonal elements are set to $1 /\left(2 m_{0}\right)$. Again, $m_{0}$ is the mass of the nucleus and $m_{1}, \ldots, m_{n}$ are the electron masses. Next we define

$$
\lambda=\operatorname{tr}\left[A_{k l}^{-1} J\right],
$$

common only to the potential energy matrix element, where matrix $J$ has the following simple structure:

$$
J=\left\{\begin{array}{ll}
E_{i i}, & i=j \text { for } r_{i} \\
E_{i i}+E_{j j}-E_{i j}-E_{j i}, & i \neq j \text { for } r_{i j}
\end{array},\right.
$$

and $E_{i j}$ is a matrix with 1 in the $i, j$ th position and 0 's elsewhere.

In addition to these quatities we also define operations used to determine the gradient formulas. First, the "vech" operation transforms an $n \times n$ matrix into an $n(n+$ 1)/2-component vector and, second, the transformation matrix, $\mathcal{T}$, formed by the first derivatives of the elements of vech of a $3 n \times 3 n$ matrix $\mathbf{L}_{k}\left(\mathbf{L}_{l}\right)$ with respect to the elements of vech of $n \times n$ matrix $L_{k}\left(L_{l}\right)$. $\mathcal{T}$ is defined as

$$
\mathcal{T}=\frac{\mathrm{d} \text { vech } \mathbf{L}_{k}\left(\operatorname{vech} L_{k}\right)}{\mathrm{d}\left(\operatorname{vech} L_{k}\right)^{\prime}} \equiv \frac{\mathrm{d} \operatorname{vech} \mathbf{L}_{l}\left(\operatorname{vech} L_{l}\right)}{\mathrm{d}\left(\operatorname{vech} L_{l}\right)^{\prime}}
$$

The overlap integral for $\phi_{k}$ and $\phi_{l}$ basis functions is

$$
\left\langle\phi_{k} \mid \phi_{l}\right\rangle=\frac{1}{2} \pi^{3 n / 2}\left|A_{k l}\right|^{-3 / 2} \eta,
$$

and its derivatives with respect to vech $L_{k}$ and vech $L_{l}$ are

$$
\begin{aligned}
\frac{\partial\left\langle\phi_{k} \mid \phi_{l}\right\rangle}{\partial \operatorname{vech} L_{k}}= & -\frac{1}{2} \pi^{3 n / 2}\left|A_{k l}^{-1}\right|^{3 / 2}\left\{\frac{3}{2} \operatorname{vech}\left(\left(A_{k l}^{-1}+A_{k l}^{-1 \prime}\right) L_{k}\right) \eta\right. \\
& +\operatorname{vech}\left(\left(\mathbf{A}_{k l}^{-1} \mathbf{W}_{k} \mathbf{A}_{k l}^{-1} \mathbf{W}_{l} \mathbf{A}_{k l}^{-1}+\left(\mathbf{A}_{k l}^{-1} \mathbf{W}_{k} \mathbf{A}_{k l}^{-1} \mathbf{W}_{l} \mathbf{A}_{k l}^{-1}\right)^{\prime}\right) \mathbf{L}_{k}\right) \mathcal{T} \\
& \left.+\operatorname{vech}\left(\left(\mathbf{A}_{k l}^{-1} \mathbf{W}_{l} \mathbf{A}_{k l}^{-1} \mathbf{W}_{k} \mathbf{A}_{k l}^{-1}+\left(\mathbf{A}_{k l}^{-1} \mathbf{W}_{l} \mathbf{A}_{k l}^{-1} \mathbf{W}_{k} \mathbf{A}_{k l}^{-1}\right)^{\prime}\right) \mathbf{L}_{k}\right) \mathcal{T}\right\}, \\
\frac{\partial\left\langle\phi_{k} \mid \phi_{l}\right\rangle}{\partial \operatorname{vech} L_{l}=} & -\frac{1}{2} \pi^{3 n / 2}\left|A_{k l}^{-1}\right|^{3 / 2}\left\{\frac{3}{2} \operatorname{vech}\left(\left(A_{k l}^{-1}+A_{k l}^{-1 \prime}\right) L_{l}\right) \eta\right. \\
& +\operatorname{vech}\left(\left(\mathbf{A}_{k l}^{-1} \mathbf{W}_{k} \mathbf{A}_{k l}^{-1} \mathbf{W}_{l} \mathbf{A}_{k l}^{-1}+\left(\mathbf{A}_{k l}^{-1} \mathbf{W}_{k} \mathbf{A}_{k l}^{-1} \mathbf{W}_{l} \mathbf{A}_{k l}^{-1}\right)^{\prime}\right) \mathbf{L}_{l}\right) \mathcal{T} \\
& \left.+\operatorname{vech}\left(\left(\mathbf{A}_{k l}^{-1} \mathbf{W}_{l} \mathbf{A}_{k l}^{-1} \mathbf{W}_{k} \mathbf{A}_{k l}^{-1}+\left(\mathbf{A}_{k l}^{-1} \mathbf{W}_{l} \mathbf{A}_{k l}^{-1} \mathbf{W}_{k} \mathbf{A}_{k l}^{-1}\right)^{\prime}\right) \mathbf{L}_{l}\right) \mathcal{T}\right\} .
\end{aligned}
$$


The kinetic energy matrix element is

$$
\begin{aligned}
\mathrm{T}_{k l}=\left\langle\phi_{k}\left|-\nabla_{\mathbf{r}}^{\prime} \mathbf{M} \nabla_{\mathbf{r}}^{\prime}\right| \phi_{l}\right\rangle= & \pi^{3 n / 2}\left|A_{k l}\right|^{-3 / 2}\left\{3 \eta \tau+2\left(\operatorname{tr}\left[\mathbf{A}_{k l}^{-1} \mathbf{W}_{k} \mathbf{A}_{k l}^{-1} \mathbf{W}_{l} \mathbf{A}_{k l}^{-1} \mathbf{A}_{k} \mathbf{M} \mathbf{A}_{l}\right]+\operatorname{tr}\left[\mathbf{A}_{k l}^{-1} \mathbf{W}_{k} \mathbf{M} \mathbf{W}_{l}\right]\right.\right. \\
& -\operatorname{tr}\left[\mathbf{A}_{k l}^{-1} \mathbf{W}_{k} \mathbf{A}_{k l}^{-1} \mathbf{A}_{k} \mathbf{M} \mathbf{W}_{l}\right]-\operatorname{tr}\left[\mathbf{A}_{k l}^{-1} \mathbf{W}_{l} \mathbf{A}_{k l}^{-1} \mathbf{W}_{k} \mathbf{M} \mathbf{A}_{l}\right] \\
& \left.\left.+\operatorname{tr}\left[\mathbf{A}_{k l}^{-1} \mathbf{W}_{l} \mathbf{A}_{k l}^{-1} \mathbf{W}_{k} \mathbf{A}_{k l}^{-1} \mathbf{A}_{k} \mathbf{M} \mathbf{A}_{l}\right]\right)\right\}
\end{aligned}
$$

and its derivative with respect to vech $L_{k}$ and vech $L_{l}$ are

$$
\begin{aligned}
& \frac{\partial \mathrm{T}_{k l}}{\partial \operatorname{vech} L_{k}}=\frac{\partial\left\langle\phi_{k}\left|-\nabla_{\mathbf{r}}^{\prime} \mathbf{M} \nabla_{\mathbf{r}}^{\prime}\right| \phi_{l}\right\rangle}{\partial \operatorname{vech}\left(L_{k}\right)} \\
& =-\pi^{3 n / 2}\left|A_{k l}\right|^{-3 / 2}\left\{\frac { 3 } { 2 } \operatorname { v e c h } ( ( A _ { k l } ^ { - 1 } + A _ { k l } ^ { - 1 \prime } ) L _ { k } ) \left(3 \eta \tau+2\left(\operatorname{tr}\left[\mathbf{A}_{k l}^{-1} \mathbf{W}_{k} \mathbf{A}_{k l}^{-1} \mathbf{W}_{l} \mathbf{A}_{k l}^{-1} \mathbf{A}_{k} \mathbf{M} \mathbf{A}_{l}\right]+\operatorname{tr}\left[\mathbf{A}_{k l}^{-1} \mathbf{W}_{k} \mathbf{M} \mathbf{W}_{l}\right]\right.\right.\right. \\
& +\operatorname{tr}\left[\mathbf{A}_{k l}^{-1} \mathbf{W}_{k} \mathbf{A}_{k l}^{-1} \mathbf{A}_{k} \mathbf{M} \mathbf{W}_{l}\right]+\operatorname{tr}\left[\mathbf{A}_{k l}^{-1} \mathbf{W}_{l} \mathbf{A}_{k l}^{-1} \mathbf{W}_{k} \mathbf{M} \mathbf{A}_{l}\right] \\
& \left.\left.+\operatorname{tr}\left[\mathbf{A}_{k l}^{-1} \mathbf{W}_{l} \mathbf{A}_{k l}^{-1} \mathbf{W}_{k} \mathbf{A}_{k l}^{-1} \mathbf{A}_{k} \mathbf{M} \mathbf{A}_{l}\right]\right)\right) \\
& +3 \tau\left(\operatorname{vech}\left(\left(\mathbf{A}_{k l}^{-1} \mathbf{W}_{k} \mathbf{A}_{k l}^{-1} \mathbf{W}_{l} \mathbf{A}_{k l}^{-1}+\left(\mathbf{A}_{k l}^{-1} \mathbf{W}_{k} \mathbf{A}_{k l}^{-1} \mathbf{W}_{l} \mathbf{A}_{k l}^{-1}\right)^{\prime}\right) \mathbf{L}_{k}\right)\right. \\
& \left.+\operatorname{vech}\left(\left(\mathbf{A}_{k l}^{-1} \mathbf{W}_{l} \mathbf{A}_{k l}^{-1} \mathbf{W}_{k} \mathbf{A}_{k l}^{-1}+\left(\mathbf{A}_{k l}^{-1} \mathbf{W}_{l} \mathbf{A}_{k l}^{-1} \mathbf{W}_{k} \mathbf{A}_{k l}^{-1}\right)^{\prime}\right) \mathbf{L}_{k}\right)\right) \mathcal{T} \\
& +3 \eta\left(\operatorname{vech}\left(\left(A_{k l}^{-1} A_{k} M A_{l} A_{k l}^{-1}+\left(A_{k l}^{-1} A_{k} M A_{l} A_{k l}^{-1}\right)^{\prime}\right) L_{k}\right)\right. \\
& \left.-\operatorname{vech}\left(\left(M A_{l} A_{k l}^{-1}+\left(M A_{l} A_{k l}^{-1}\right)^{\prime}\right) L_{k}\right)\right) \\
& +2\left(\operatorname{vech}\left(\left(\mathbf{A}_{k l}^{-1} \mathbf{W}_{k} \mathbf{A}_{k l}^{-1} \mathbf{W}_{l} \mathbf{A}_{k l}^{-1} \mathbf{A}_{k} \mathbf{M} \mathbf{A}_{l} \mathbf{A}_{k l}^{-1}+\left(\mathbf{A}_{k l}^{-1} \mathbf{W}_{k} \mathbf{A}_{k l}^{-1} \mathbf{W}_{l} \mathbf{A}_{k l}^{-1} \mathbf{A}_{k} \mathbf{M} \mathbf{A}_{l} \mathbf{A}_{k l}^{-1}\right)^{\prime}\right) \mathbf{L}_{k}\right)\right. \\
& +\operatorname{vech}\left(\left(\mathbf{A}_{k l}^{-1} \mathbf{W}_{l} \mathbf{A}_{k l}^{-1} \mathbf{A}_{k} \mathbf{M} \mathbf{A}_{l} \mathbf{A}_{k l}^{-1} \mathbf{W}_{k} \mathbf{A}_{k l}^{-1}+\left(\mathbf{A}_{k l}^{-1} \mathbf{W}_{l} \mathbf{A}_{k l}^{-1} \mathbf{A}_{k} \mathbf{M} \mathbf{A}_{l} \mathbf{A}_{k l}^{-1} \mathbf{W}_{k} \mathbf{A}_{k l}^{-1}\right)^{\prime}\right) \mathbf{L}_{k}\right) \\
& +\operatorname{vech}\left(\left(\mathbf{A}_{k l}^{-1} \mathbf{A}_{k} \mathbf{M} \mathbf{A}_{l} \mathbf{A}_{k l}^{-1} \mathbf{W}_{k} \mathbf{A}_{k l}^{-1} \mathbf{W}_{l} \mathbf{A}_{k l}^{-1}+\left(\mathbf{A}_{k l}^{-1} \mathbf{A}_{k} \mathbf{M} \mathbf{A}_{l} \mathbf{A}_{k l}^{-1} \mathbf{W}_{k} \mathbf{A}_{k l}^{-1} \mathbf{W}_{l} \mathbf{A}_{k l}^{-1}\right)^{\prime}\right) \mathbf{L}_{k}\right) \\
& -\operatorname{vech}\left(\left(\mathbf{M} \mathbf{A}_{l} \mathbf{A}_{k l}^{-1} \mathbf{W}_{k} \mathbf{A}_{k l}^{-1} \mathbf{W}_{l} \mathbf{A}_{k l}^{-1}+\left(\mathbf{M} \mathbf{A}_{l} \mathbf{A}_{k l}^{-1} \mathbf{W}_{k} \mathbf{A}_{k l}^{-1} \mathbf{W}_{l} \mathbf{A}_{k l}^{-1}\right)^{\prime}\right) \mathbf{L}_{k}\right) \\
& +\operatorname{vech}\left(\left(\mathbf{A}_{k l}^{-1} \mathbf{W}_{l} \mathbf{A}_{k l}^{-1} \mathbf{W}_{k} \mathbf{A}_{k l}^{-1} \mathbf{A}_{k} \mathbf{M} \mathbf{A}_{l} \mathbf{A}_{k l}^{-1}+\left(\mathbf{A}_{k l}^{-1} \mathbf{W}_{l} \mathbf{A}_{k l}^{-1} \mathbf{W}_{k} \mathbf{A}_{k l}^{-1} \mathbf{A}_{k} \mathbf{M} \mathbf{A}_{l} \mathbf{A}_{k l}^{-1}\right)^{\prime}\right) \mathbf{L}_{k}\right) \\
& +\operatorname{vech}\left(\left(\mathbf{A}_{k l}^{-1} \mathbf{W}_{k} \mathbf{A}_{k l}^{-1} \mathbf{A}_{k} \mathbf{M} \mathbf{A}_{l} \mathbf{A}_{k l}^{-1} \mathbf{W}_{l} \mathbf{A}_{k l}^{-1}+\left(\mathbf{A}_{k l}^{-1} \mathbf{W}_{k} \mathbf{A}_{k l}^{-1} \mathbf{A}_{k} \mathbf{M} \mathbf{A}_{l} \mathbf{A}_{k l}^{-1} \mathbf{W}_{l} \mathbf{A}_{k l}^{-1}\right)^{\prime}\right) \mathbf{L}_{k}\right) \\
& +\operatorname{vech}\left(\left(\mathbf{A}_{k l}^{-1} \mathbf{A}_{k} \mathbf{M} \mathbf{A}_{l} \mathbf{A}_{k l}^{-1} \mathbf{W}_{l} \mathbf{A}_{k l}^{-1} \mathbf{W}_{k} \mathbf{A}_{k l}^{-1}+\left(\mathbf{A}_{k l}^{-1} \mathbf{A}_{k} \mathbf{M} \mathbf{A}_{l} \mathbf{A}_{k l}^{-1} \mathbf{W}_{l} \mathbf{A}_{k l}^{-1} \mathbf{W}_{k} \mathbf{A}_{k l}^{-1}\right)^{\prime}\right) \mathbf{L}_{k}\right) \\
& -\operatorname{vech}\left(\left(\mathbf{M} \mathbf{A}_{l} \mathbf{A}_{k l}^{-1} \mathbf{W}_{l} \mathbf{A}_{k l}^{-1} \mathbf{W}_{k} \mathbf{A}_{k l}^{-1}+\left(\mathbf{M} \mathbf{A}_{l} \mathbf{A}_{k l}^{-1} \mathbf{W}_{l} \mathbf{A}_{k l}^{-1} \mathbf{W}_{k} \mathbf{A}_{k l}^{-1}\right)^{\prime}\right) \mathbf{L}_{k}\right) \\
& +\operatorname{vech}\left(\left(\mathbf{A}_{k l}^{-1} \mathbf{W}_{k} \mathbf{A}_{k l}^{-1} \mathbf{A}_{k} \mathbf{M} \mathbf{W}_{l} \mathbf{A}_{k l}^{-1}+\left(\mathbf{A}_{k l}^{-1} \mathbf{W}_{k} \mathbf{A}_{k l}^{-1} \mathbf{A}_{k} \mathbf{M} \mathbf{W}_{l} \mathbf{A}_{k l}^{-1}\right)^{\prime}\right) \mathbf{L}_{k}\right) \\
& +\operatorname{vech}\left(\left(\mathbf{A}_{k l}^{-1} \mathbf{A}_{k} \mathbf{M} \mathbf{W}_{l} \mathbf{A}_{k l}^{-1} \mathbf{W}_{k} \mathbf{A}_{k l}^{-1}+\left(\mathbf{A}_{k l}^{-1} \mathbf{A}_{k} \mathbf{M} \mathbf{W}_{l} \mathbf{A}_{k l}^{-1} \mathbf{W}_{k} \mathbf{A}_{k l}^{-1}\right)^{\prime}\right) \mathbf{L}_{k}\right) \\
& -\operatorname{vech}\left(\left(\mathbf{M W}_{l} \mathbf{A}_{k l}^{-1} \mathbf{W}_{k} \mathbf{A}_{k l}^{-1}+\left(\mathbf{M} \mathbf{W}_{l} \mathbf{A}_{k l}^{-1} \mathbf{W}_{k} \mathbf{A}_{k l}^{-1}\right)^{\prime}\right) \mathbf{L}_{k}\right) \\
& +\operatorname{vech}\left(\left(\mathbf{A}_{k l}^{-1} \mathbf{W}_{l} \mathbf{A}_{k l}^{-1} \mathbf{W}_{k} \mathbf{M} \mathbf{A}_{l} \mathbf{A}_{k l}^{-1}+\left(\mathbf{A}_{k l}^{-1} \mathbf{W}_{l} \mathbf{A}_{k l}^{-1} \mathbf{W}_{k} \mathbf{M} \mathbf{A}_{l} \mathbf{A}_{k l}^{-1}\right)^{\prime}\right) \mathbf{L}_{k}\right) \\
& +\operatorname{vech}\left(\left(\mathbf{A}_{k l}^{-1} \mathbf{W}_{k} \mathbf{M} \mathbf{A}_{l} \mathbf{A}_{k l}^{-1} \mathbf{W}_{l} \mathbf{A}_{k l}^{-1}+\left(\mathbf{A}_{k l}^{-1} \mathbf{W}_{k} \mathbf{M} \mathbf{A}_{l} \mathbf{A}_{k l}^{-1} \mathbf{W}_{l} \mathbf{A}_{k l}^{-1}\right)^{\prime}\right) \mathbf{L}_{k}\right) \\
& \left.\left.+\operatorname{vech}\left(\left(\mathbf{A}_{k l}^{-1} \mathbf{W}_{k} \mathbf{M} \mathbf{W}_{l} \mathbf{A}_{k l}^{-1}+\left(\mathbf{A}_{k l}^{-1} \mathbf{W}_{k} \mathbf{M} \mathbf{W}_{l} \mathbf{A}_{k l}^{-1}\right)^{\prime}\right) \mathbf{L}_{k}\right)\right) \mathcal{T}\right\}
\end{aligned}
$$




$$
\begin{aligned}
& \frac{\partial \mathbf{T}_{k l}}{\partial \operatorname{vech} L_{l}}=\frac{\partial\left\langle\phi_{k}\left|-\nabla_{\mathbf{r}}^{\prime} \mathbf{M} \nabla_{\mathbf{r}}^{\prime}\right| \phi_{l}\right\rangle}{\partial \operatorname{vech} L_{l}} \\
& =-\pi^{3 n / 2}\left|A_{k l}\right|^{-3 / 2}\left\{\frac { 3 } { 2 } \operatorname { v e c h } ( ( A _ { k l } ^ { - 1 } + A _ { k l } ^ { - 1 \prime } ) L _ { l } ) \left(\eta \tau+2\left(\operatorname{tr}\left[\mathbf{A}_{k l}^{-1} \mathbf{W}_{k} \mathbf{A}_{k l}^{-1} \mathbf{W}_{l} \mathbf{A}_{k l}^{-1} \mathbf{A}_{k} \mathbf{M} \mathbf{A}_{l}\right]+\operatorname{tr}\left[\mathbf{A}_{k l}^{-1} \mathbf{W}_{k} \mathbf{M} \mathbf{W}_{l}\right]\right.\right.\right. \\
& \left.\left.+\operatorname{tr}\left[\mathbf{A}_{k l}^{-1} \mathbf{W}_{k} \mathbf{A}_{k l}^{-1} \mathbf{A}_{k} \mathbf{M} \mathbf{W}_{l}\right]+\operatorname{tr}\left[\mathbf{A}_{k l}^{-1} \mathbf{W}_{l} \mathbf{A}_{k l}^{-1} \mathbf{W}_{k} \mathbf{M} \mathbf{A}_{l}\right]+\operatorname{tr}\left[\mathbf{A}_{k l}^{-1} \mathbf{W}_{l} \mathbf{A}_{k l}^{-1} \mathbf{W}_{k} \mathbf{A}_{k l}^{-1} \mathbf{A}_{k} \mathbf{M} \mathbf{A}_{l}\right]\right)\right) \\
& +3 \tau\left(\operatorname{vech}\left(\left(\mathbf{A}_{k l}^{-1} \mathbf{W}_{k} \mathbf{A}_{k l}^{-1} \mathbf{W}_{l} \mathbf{A}_{k l}^{-1}+\left(\mathbf{A}_{k l}^{-1} \mathbf{W}_{k} \mathbf{A}_{k l}^{-1} \mathbf{W}_{l} \mathbf{A}_{k l}^{-1}\right)^{\prime}\right) \mathbf{L}_{l}\right)\right. \\
& \left.+\operatorname{vech}\left(\left(\mathbf{A}_{k l}^{-1} \mathbf{W}_{l} \mathbf{A}_{k l}^{-1} \mathbf{W}_{k} \mathbf{A}_{k l}^{-1}+\left(\mathbf{A}_{k l}^{-1} \mathbf{W}_{l} \mathbf{A}_{k l}^{-1} \mathbf{W}_{k} \mathbf{A}_{k l}^{-1}\right)^{\prime}\right) \mathbf{L}_{k}\right)\right) \mathcal{T} \\
& +3 \eta\left(\operatorname{vech}\left(\left(A_{k l}^{-1} A_{k} M A_{l} A_{k l}^{-1}+\left(A_{k l}^{-1} A_{k} M A_{l} A_{k l}^{-1}\right)^{\prime}\right) L_{l}\right)\right. \\
& \text { - vech } \left.\left(\left(A_{k l}^{-1} A_{k} M+\left(A_{k l}^{-1} A_{k} M\right)^{\prime}\right) L_{l}\right)\right) \\
& +2\left(\operatorname{vech}\left(\left(\mathbf{A}_{k l}^{-1} \mathbf{W}_{k} \mathbf{A}_{k l}^{-1} \mathbf{W}_{l} \mathbf{A}_{k l}^{-1} \mathbf{A}_{k} \mathbf{M} \mathbf{A}_{l} \mathbf{A}_{k l}^{-1}+\left(\mathbf{A}_{k l}^{-1} \mathbf{W}_{k} \mathbf{A}_{k l}^{-1} \mathbf{W}_{l} \mathbf{A}_{k l}^{-1} \mathbf{A}_{k} \mathbf{M} \mathbf{A}_{l} \mathbf{A}_{k l}^{-1}\right)^{\prime}\right) \mathbf{L}_{l}\right)\right. \\
& +\operatorname{vech}\left(\left(\mathbf{A}_{k l}^{-1} \mathbf{W}_{l} \mathbf{A}_{k l}^{-1} \mathbf{A}_{k} \mathbf{M} \mathbf{A}_{l} \mathbf{A}_{k l}^{-1} \mathbf{W}_{k} \mathbf{A}_{k l}^{-1}+\left(\mathbf{A}_{k l}^{-1} \mathbf{W}_{l} \mathbf{A}_{k l}^{-1} \mathbf{A}_{k} \mathbf{M} \mathbf{A}_{l} \mathbf{A}_{k l}^{-1} \mathbf{W}_{k} \mathbf{A}_{k l}^{-1}\right)^{\prime}\right) \mathbf{L}_{l}\right) \\
& +\operatorname{vech}\left(\left(\mathbf{A}_{k l}^{-1} \mathbf{A}_{k} \mathbf{M} \mathbf{A}_{l} \mathbf{A}_{k l}^{-1} \mathbf{W}_{k} \mathbf{A}_{k l}^{-1} \mathbf{W}_{l} \mathbf{A}_{k l}^{-1}+\left(\mathbf{A}_{k l}^{-1} \mathbf{A}_{k} \mathbf{M} \mathbf{A}_{l} \mathbf{A}_{k l}^{-1} \mathbf{W}_{k} \mathbf{A}_{k l}^{-1} \mathbf{W}_{l} \mathbf{A}_{k l}^{-1}\right)^{\prime}\right) \mathbf{L}_{l}\right) \\
& -\operatorname{vech}\left(\left(\mathbf{A}_{k l}^{-1} \mathbf{W}_{k} \mathbf{A}_{k l}^{-1} \mathbf{W}_{l} \mathbf{A}_{k l}^{-1} \mathbf{A}_{k} \mathbf{M}+\left(\mathbf{A}_{k l}^{-1} \mathbf{W}_{k} \mathbf{A}_{k l}^{-1} \mathbf{W}_{l} \mathbf{A}_{k l}^{-1} \mathbf{A}_{k} \mathbf{M}\right)^{\prime}\right) \mathbf{L}_{l}\right) \\
& +\operatorname{vech}\left(\left(\mathbf{A}_{k l}^{-1} \mathbf{W}_{l} \mathbf{A}_{k l}^{-1} \mathbf{W}_{k} \mathbf{A}_{k l}^{-1} \mathbf{A}_{k} \mathbf{M} \mathbf{A}_{l} \mathbf{A}_{k l}^{-1}+\left(\mathbf{A}_{k l}^{-1} \mathbf{W}_{l} \mathbf{A}_{k l}^{-1} \mathbf{W}_{k} \mathbf{A}_{k l}^{-1} \mathbf{A}_{k} \mathbf{M} \mathbf{A}_{l} \mathbf{A}_{k l}^{-1}\right)^{\prime}\right) \mathbf{L}_{l}\right) \\
& +\operatorname{vech}\left(\left(\mathbf{A}_{k l}^{-1} \mathbf{W}_{k} \mathbf{A}_{k l}^{-1} \mathbf{A}_{k} \mathbf{M} \mathbf{A}_{l} \mathbf{A}_{k l}^{-1} \mathbf{W}_{l} \mathbf{A}_{k l}^{-1}+\left(\mathbf{A}_{k l}^{-1} \mathbf{W}_{k} \mathbf{A}_{k l}^{-1} \mathbf{A}_{k} \mathbf{M} \mathbf{A}_{l} \mathbf{A}_{k l}^{-1} \mathbf{W}_{l} \mathbf{A}_{k l}^{-1}\right)^{\prime}\right) \mathbf{L}_{l}\right) \\
& +\operatorname{vech}\left(\left(\mathbf{A}_{k l}^{-1} \mathbf{A}_{k} \mathbf{M} \mathbf{A}_{l} \mathbf{A}_{k l}^{-1} \mathbf{W}_{l} \mathbf{A}_{k l}^{-1} \mathbf{W}_{k} \mathbf{A}_{k l}^{-1}+\left(\mathbf{A}_{k l}^{-1} \mathbf{A}_{k} \mathbf{M} \mathbf{A}_{l} \mathbf{A}_{k l}^{-1} \mathbf{W}_{l} \mathbf{A}_{k l}^{-1} \mathbf{W}_{k} \mathbf{A}_{k l}^{-1}\right)^{\prime}\right) \mathbf{L}_{l}\right) \\
& -\operatorname{vech}\left(\left(\mathbf{A}_{k l}^{-1} \mathbf{W}_{l} \mathbf{A}_{k l}^{-1} \mathbf{W}_{k} \mathbf{A}_{k l}^{-1} \mathbf{A}_{k} \mathbf{M}+\left(\mathbf{A}_{k l}^{-1} \mathbf{W}_{l} \mathbf{A}_{k l}^{-1} \mathbf{W}_{k} \mathbf{A}_{k l}^{-1} \mathbf{A}_{k} \mathbf{M}\right)^{\prime}\right) \mathbf{L}_{l}\right) \\
& +\operatorname{vech}\left(\left(\mathbf{A}_{k l}^{-1} \mathbf{W}_{k} \mathbf{A}_{k l}^{-1} \mathbf{A}_{k} \mathbf{M} \mathbf{W}_{l} \mathbf{A}_{k l}^{-1}+\left(\mathbf{A}_{k l}^{-1} \mathbf{W}_{k} \mathbf{A}_{k l}^{-1} \mathbf{A}_{k} \mathbf{M} \mathbf{W}_{l} \mathbf{A}_{k l}^{-1}\right)^{\prime}\right) \mathbf{L}_{l}\right) \\
& +\operatorname{vech}\left(\left(\mathbf{A}_{k l}^{-1} \mathbf{A}_{k} \mathbf{M} \mathbf{W}_{l} \mathbf{A}_{k l}^{-1} \mathbf{W}_{k} \mathbf{A}_{k l}^{-1}+\left(\mathbf{A}_{k l}^{-1} \mathbf{A}_{k} \mathbf{M} \mathbf{W}_{l} \mathbf{A}_{k l}^{-1} \mathbf{W}_{k} \mathbf{A}_{k l}^{-1}\right)^{\prime}\right) \mathbf{L}_{l}\right) \\
& +\operatorname{vech}\left(\left(\mathbf{A}_{k l}^{-1} \mathbf{W}_{l} \mathbf{A}_{k l}^{-1} \mathbf{W}_{k} \mathbf{M} \mathbf{A}_{l} \mathbf{A}_{k l}^{-1}+\left(\mathbf{A}_{k l}^{-1} \mathbf{W}_{l} \mathbf{A}_{k l}^{-1} \mathbf{W}_{k} \mathbf{M} \mathbf{A}_{l} \mathbf{A}_{k l}^{-1}\right)^{\prime}\right) \mathbf{L}_{l}\right) \\
& +\operatorname{vech}\left(\left(\mathbf{A}_{k l}^{-1} \mathbf{W}_{k} \mathbf{M} \mathbf{A}_{l} \mathbf{A}_{k l}^{-1} \mathbf{W}_{l} \mathbf{A}_{k l}^{-1}+\left(\mathbf{A}_{k l}^{-1} \mathbf{W}_{k} \mathbf{M} \mathbf{A}_{l} \mathbf{A}_{k l}^{-1} \mathbf{W}_{l} \mathbf{A}_{k l}^{-1}\right)^{\prime}\right) \mathbf{L}_{l}\right) \\
& -\operatorname{vech}\left(\left(\mathbf{A}_{k l}^{-1} \mathbf{W}_{l} \mathbf{A}_{k l}^{-1} \mathbf{W}_{k} \mathbf{M}+\left(\mathbf{A}_{k l}^{-1} \mathbf{W}_{l} \mathbf{A}_{k l}^{-1} \mathbf{W}_{k} \mathbf{M}\right)^{\prime}\right) \mathbf{L}_{l}\right) \\
& \left.\left.+\operatorname{vech}\left(\left(\mathbf{A}_{k l}^{-1} \mathbf{W}_{k} \mathbf{M} \mathbf{W}_{l} \mathbf{A}_{k l}^{-1}+\left(\mathbf{A}_{k l}^{-1} \mathbf{W}_{k} \mathbf{M} \mathbf{W}_{l} \mathbf{A}_{k l}^{-1}\right)^{\prime}\right) \mathbf{L}_{l}\right)\right) \mathcal{T}\right\} .
\end{aligned}
$$

And finally, the potential energy matrix element is

$$
\begin{aligned}
\mathbf{V}_{k l}=\left\langle\phi_{k}\left|\frac{1}{r_{i j}}\right| \phi_{l}\right\rangle= & 2 \pi^{(3 n-1) / 2}\left|A_{k l}\right|^{-3 / 2} \lambda^{-1 / 2}\left\{\frac{1}{2} \eta_{-} \frac{1}{6} \lambda^{-1}\left(\operatorname{tr}\left[\mathbf{A}_{k l}^{-1} \mathbf{W}_{k} \mathbf{A}_{k l}^{-1} \mathbf{W}_{l} \mathbf{A}_{k l}^{-1} \mathbf{J}\right]+\operatorname{tr}\left[\mathbf{A}_{k l}^{-1} \mathbf{W}_{l} \mathbf{A}_{k l}^{-1} \mathbf{W}_{k} \mathbf{A}_{k l}^{-1} \mathbf{J}\right]\right)\right. \\
& \left.+\frac{1}{20} \lambda^{-2}\left(2 \operatorname{tr}\left[\mathbf{A}_{k l}^{-1} \mathbf{W}_{k} \mathbf{A}_{k l}^{-1} \mathbf{J} \mathbf{A}_{k l}^{-1} \mathbf{W}_{l} \mathbf{A}_{k l}^{-1} \mathbf{J}\right]+\operatorname{tr}\left[\mathbf{A}_{k l}^{-1} \mathbf{W}_{k} \mathbf{A}_{k l}^{-1} \mathbf{J}\right] \operatorname{tr}\left[\mathbf{A}_{k l}^{-1} \mathbf{W}_{l} \mathbf{A}_{k l}^{-1} \mathbf{J}\right]\right)\right\}
\end{aligned}
$$

and its derivatives with respect to vech $L_{k}$ and vech $L_{l}$ are

$$
\begin{aligned}
\frac{\partial \mathrm{V}_{k l}}{\partial \operatorname{vech} L_{k}} & =\frac{\partial\left\langle\phi_{k}\left|\frac{1}{r_{i j}}\right| \phi_{l}\right\rangle}{\partial \operatorname{vech} L_{k}} \\
& =2 \pi^{(3 n-1) / 2}\left|A_{k l}\right|^{-3 / 2} \lambda^{-1 / 2}\left\{\left(\frac{1}{2} \lambda^{-1} \operatorname{vech}\left(\left(A_{k l}^{-1} J A_{k l}^{-1}+\left(A_{k l}^{-1} J A_{k l}^{-1}\right)^{\prime}\right) L_{k}\right)-\frac{3}{2} \operatorname{vech}\left(\left(A_{k l}^{-1}+A_{k l}^{-1 \prime}\right) L_{k}\right)\right)\right.
\end{aligned}
$$




$$
\begin{aligned}
& \times\left(\frac{1}{2} \eta-\frac{1}{6} \lambda^{-1}\left(\operatorname{tr}\left[\mathbf{A}_{k l}^{-1} \mathbf{W}_{k} \mathbf{A}_{k l}^{-1} \mathbf{W}_{l} \mathbf{A}_{k l}^{-1} \mathbf{J}\right]+\operatorname{tr}\left[\mathbf{A}_{k l}^{-1} \mathbf{W}_{l} \mathbf{A}_{k l}^{-1} \mathbf{W}_{k} \mathbf{A}_{k l}^{-1} \mathbf{J}\right]\right)\right) \\
& +\frac{1}{2}\left(\operatorname{vech}\left(\left(\mathbf{A}_{k l}^{-1} \mathbf{W}_{k} \mathbf{A}_{k l}^{-1} \mathbf{W}_{l} \mathbf{A}_{k l}^{-1}+\left(\mathbf{A}_{k l}^{-1} \mathbf{W}_{k} \mathbf{A}_{k l}^{-1} \mathbf{W}_{l} \mathbf{A}_{k l}^{-1}\right)^{\prime}\right) \mathbf{L}_{k}\right)\right. \\
& \left.+\operatorname{vech}\left(\left(\mathbf{A}_{k l}^{-1} \mathbf{W}_{l} \mathbf{A}_{k l}^{-1} \mathbf{W}_{k} \mathbf{A}_{k l}^{-1}+\left(\mathbf{A}_{k l}^{-1} \mathbf{W}_{l} \mathbf{A}_{k l}^{-1} \mathbf{W}_{k} \mathbf{A}_{k l}^{-1}\right)^{\prime}\right) \mathbf{L}_{k}\right)\right) \mathcal{T} \\
& +\frac{1}{6} \lambda^{-1}\left(\operatorname{vech}\left(\left(\mathbf{A}_{k l}^{-1} \mathbf{W}_{k} \mathbf{A}_{k l}^{-1} \mathbf{W}_{l} \mathbf{A}_{k l}^{-1} \mathbf{J} \mathbf{A}_{k l}^{-1}+\left(\mathbf{A}_{k l}^{-1} \mathbf{W}_{k} \mathbf{A}_{k l}^{-1} \mathbf{W}_{l} \mathbf{A}_{k l}^{-1} \mathbf{J} \mathbf{A}_{k l}^{-1}\right)^{\prime}\right) \mathbf{L}_{k}\right)\right. \\
& +\operatorname{vech}\left(\left(\mathbf{A}_{k l}^{-1} \mathbf{W}_{l} \mathbf{A}_{k l}^{-1} \mathbf{J} \mathbf{A}_{k l}^{-1} \mathbf{W}_{k} \mathbf{A}_{k l}^{-1}+\left(\mathbf{A}_{k l}^{-1} \mathbf{W}_{l} \mathbf{A}_{k l}^{-1} \mathbf{J} \mathbf{A}_{k l}^{-1} \mathbf{W}_{k} \mathbf{A}_{k l}^{-1}\right)^{\prime}\right) \mathbf{L}_{k}\right) \\
& +\operatorname{vech}\left(\left(\mathbf{A}_{k l}^{-1} \mathbf{J} \mathbf{A}_{k l}^{-1} \mathbf{W}_{k} \mathbf{A}_{k l}^{-1} \mathbf{W}_{l} \mathbf{A}_{k l}^{-1}+\left(\mathbf{A}_{k l}^{-1} \mathbf{J} \mathbf{A}_{k l}^{-1} \mathbf{W}_{k} \mathbf{A}_{k l}^{-1} \mathbf{W}_{l} \mathbf{A}_{k l}^{-1}\right)^{\prime}\right) \mathbf{L}_{k}\right) \\
& +\operatorname{vech}\left(\left(\mathbf{A}_{k l}^{-1} \mathbf{W}_{l} \mathbf{A}_{k l}^{-1} \mathbf{W}_{k} \mathbf{A}_{k l}^{-1} \mathbf{J} \mathbf{A}_{k l}^{-1}+\left(\mathbf{A}_{k l}^{-1} \mathbf{W}_{l} \mathbf{A}_{k l}^{-1} \mathbf{W}_{k} \mathbf{A}_{k l}^{-1} \mathbf{J} \mathbf{A}_{k l}^{-1}\right)^{\prime}\right) \mathbf{L}_{k}\right) \\
& +\operatorname{vech}\left(\left(\mathbf{A}_{k l}^{-1} \mathbf{W}_{k} \mathbf{A}_{k l}^{-1} \mathbf{J} \mathbf{A}_{k l}^{-1} \mathbf{W}_{l} \mathbf{A}_{k l}^{-1}+\left(\mathbf{A}_{k l}^{-1} \mathbf{W}_{k} \mathbf{A}_{k l}^{-1} \mathbf{J} \mathbf{A}_{k l}^{-1} \mathbf{W}_{l} \mathbf{A}_{k l}^{-1}\right)^{\prime}\right) \mathbf{L}_{k}\right) \\
& \left.+\operatorname{vech}\left(\left(\mathbf{A}_{k l}^{-1} \mathbf{J} \mathbf{A}_{k l}^{-1} \mathbf{W}_{l} \mathbf{A}_{k l}^{-1} \mathbf{W}_{k} \mathbf{A}_{k l}^{-1}+\left(\mathbf{A}_{k l}^{-1} \mathbf{J} \mathbf{A}_{k l}^{-1} \mathbf{W}_{l} \mathbf{A}_{k l}^{-1} \mathbf{W}_{k} \mathbf{A}_{k l}^{-1}\right)^{\prime}\right) \mathbf{L}_{k}\right)\right) \mathcal{T} \\
& +\frac{1}{6} \lambda^{-2} \operatorname{vech}\left(\left(A_{k l}^{-1} J A_{k l}^{-1}+\left(A_{k l}^{-1} J A_{k l}^{-1}\right)^{\prime}\right) L_{k}\right) \\
& \left.\left(\operatorname{tr}\left[\mathbf{A}_{k l}^{-1} \mathbf{W}_{k} \mathbf{A}_{k l}^{-1} \mathbf{W}_{l} \mathbf{A}_{k l}^{-1} \mathbf{J}\right]+\operatorname{tr}\left[\mathbf{A}_{k l}^{-1} \mathbf{W}_{l} \mathbf{A}_{k l}^{-1} \mathbf{W}_{k} \mathbf{A}_{k l}^{-1} \mathbf{J}\right]\right)\right\}
\end{aligned}
$$

$$
\begin{aligned}
& \frac{\partial \mathrm{V}_{k l}}{\partial \operatorname{vech} L_{l}}=\frac{\partial\left\langle\phi_{k}\left|\frac{1}{r_{i j}}\right| \phi_{l}\right\rangle}{\partial \operatorname{vech} L_{l}} \\
& =2 \pi^{(3 n-1) / 2}\left|A_{k l}\right|^{-3 / 2} \lambda^{-1 / 2}\left\{\left(\frac{1}{2} \lambda^{-1} \operatorname{vech}\left(\left(A_{k l}^{-1} J A_{k l}^{-1}+\left(A_{k l}^{-1} J A_{k l}^{-1}\right)^{\prime}\right) L_{l}\right)-\frac{3}{2} \operatorname{vech}\left(\left(A_{k l}^{-1}+A_{k l}^{-1 \prime}\right) L_{l}\right)\right)\right. \\
& \times\left(\frac{1}{2} \eta-\frac{1}{6} \lambda^{-1}\left(\operatorname{tr}\left[\mathbf{A}_{k l}^{-1} \mathbf{W}_{k} \mathbf{A}_{k l}^{-1} \mathbf{W}_{l} \mathbf{A}_{k l}^{-1} \mathbf{J}\right]+\operatorname{tr}\left[\mathbf{A}_{k l}^{-1} \mathbf{W}_{l} \mathbf{A}_{k l}^{-1} \mathbf{W}_{k} \mathbf{A}_{k l}^{-1} \mathbf{J}\right]\right)\right) \\
& +\frac{1}{2}\left(\operatorname{vech}\left(\left(\mathbf{A}_{k l}^{-1} \mathbf{W}_{k} \mathbf{A}_{k l}^{-1} \mathbf{W}_{l} \mathbf{A}_{k l}^{-1}+\left(\mathbf{A}_{k l}^{-1} \mathbf{W}_{k} \mathbf{A}_{k l}^{-1} \mathbf{W}_{l} \mathbf{A}_{k l}^{-1}\right)^{\prime}\right) \mathbf{L}_{l}\right)\right. \\
& \left.+\operatorname{vech}\left(\left(\mathbf{A}_{k l}^{-1} \mathbf{W}_{l} \mathbf{A}_{k l}^{-1} \mathbf{W}_{k} \mathbf{A}_{k l}^{-1}+\left(\mathbf{A}_{k l}^{-1} \mathbf{W}_{l} \mathbf{A}_{k l}^{-1} \mathbf{W}_{k} \mathbf{A}_{k l}^{-1}\right)^{\prime}\right) \mathbf{L}_{l}\right)\right) \mathcal{T} \\
& +\frac{1}{6} \lambda^{-1}\left(\operatorname{vech}\left(\left(\mathbf{A}_{k l}^{-1} \mathbf{W}_{k} \mathbf{A}_{k l}^{-1} \mathbf{W}_{l} \mathbf{A}_{k l}^{-1} \mathbf{J} \mathbf{A}_{k l}^{-1}+\left(\mathbf{A}_{k l}^{-1} \mathbf{W}_{k} \mathbf{A}_{k l}^{-1} \mathbf{W}_{l} \mathbf{A}_{k l}^{-1} \mathbf{J} \mathbf{A}_{k l}^{-1}\right)^{\prime}\right) \mathbf{L}_{l}\right)\right. \\
& +\operatorname{vech}\left(\left(\mathbf{A}_{k l}^{-1} \mathbf{W}_{l} \mathbf{A}_{k l}^{-1} \mathbf{J} \mathbf{A}_{k l}^{-1} \mathbf{W}_{k} \mathbf{A}_{k l}^{-1}+\left(\mathbf{A}_{k l}^{-1} \mathbf{W}_{l} \mathbf{A}_{k l}^{-1} \mathbf{J A}_{k l}^{-1} \mathbf{W}_{k} \mathbf{A}_{k l}^{-1}\right)^{\prime}\right) \mathbf{L}_{l}\right) \\
& +\operatorname{vech}\left(\left(\mathbf{A}_{k l}^{-1} \mathbf{J} \mathbf{A}_{k l}^{-1} \mathbf{W}_{k} \mathbf{A}_{k l}^{-1} \mathbf{W}_{l} \mathbf{A}_{k l}^{-1}+\left(\mathbf{A}_{k l}^{-1} \mathbf{J} \mathbf{A}_{k l}^{-1} \mathbf{W}_{k} \mathbf{A}_{k l}^{-1} \mathbf{W}_{l} \mathbf{A}_{k l}^{-1}\right)^{\prime}\right) \mathbf{L}_{l}\right) \\
& +\operatorname{vech}\left(\left(\mathbf{A}_{k l}^{-1} \mathbf{J} \mathbf{A}_{k l}^{-1} \mathbf{W}_{k} \mathbf{A}_{k l}^{-1} \mathbf{W}_{l} \mathbf{A}_{k l}^{-1}+\left(\mathbf{A}_{k l}^{-1} \mathbf{J} \mathbf{A}_{k l}^{-1} \mathbf{W}_{k} \mathbf{A}_{k l}^{-1} \mathbf{W}_{l} \mathbf{A}_{k l}^{-1}\right)^{\prime}\right) \mathbf{L}_{l}\right) \\
& +\operatorname{vech}\left(\left(\mathbf{A}_{k l}^{-1} \mathbf{W}_{l} \mathbf{A}_{k l}^{-1} \mathbf{W}_{k} \mathbf{A}_{k l}^{-1} \mathbf{J} \mathbf{A}_{k l}^{-1}+\left(\mathbf{A}_{k l}^{-1} \mathbf{W}_{l} \mathbf{A}_{k l}^{-1} \mathbf{W}_{k} \mathbf{A}_{k l}^{-1} \mathbf{J A}_{k l}^{-1}\right)^{\prime}\right) \mathbf{L}_{l}\right) \\
& +\operatorname{vech}\left(\left(\mathbf{A}_{k l}^{-1} \mathbf{W}_{k} \mathbf{A}_{k l}^{-1} \mathbf{J} \mathbf{A}_{k l}^{-1} \mathbf{W}_{l} \mathbf{A}_{k l}^{-1}+\left(\mathbf{A}_{k l}^{-1} \mathbf{W}_{k} \mathbf{A}_{k l}^{-1} \mathbf{J} \mathbf{A}_{k l}^{-1} \mathbf{W}_{l} \mathbf{A}_{k l}^{-1}\right)^{\prime}\right) \mathbf{L}_{l}\right) \\
& \left.+\operatorname{vech}\left(\left(\mathbf{A}_{k l}^{-1} \mathbf{J} \mathbf{A}_{k l}^{-1} \mathbf{W}_{l} \mathbf{A}_{k l}^{-1} \mathbf{W}_{k} \mathbf{A}_{k l}^{-1}+\left(\mathbf{A}_{k l}^{-1} \mathbf{J} \mathbf{A}_{k l}^{-1} \mathbf{W}_{l} \mathbf{A}_{k l}^{-1} \mathbf{W}_{k} \mathbf{A}_{k l}^{-1}\right)^{\prime}\right) \mathbf{L}_{l}\right)\right) \mathcal{T} \\
& -\frac{1}{6} \lambda^{-2} \operatorname{vech}\left(\left(A_{k l}^{-1} J A_{k l}^{-1}+\left(A_{k l}^{-1} J A_{k l}^{-1}\right)^{\prime}\right) L_{l}\right) \\
& \left.\left(\operatorname{tr}\left[\mathbf{A}_{k l}^{-1} \mathbf{W}_{k} \mathbf{A}_{k l}^{-1} \mathbf{W}_{l} \mathbf{A}_{k l}^{-1} \mathbf{J}\right]+\operatorname{tr}\left[\mathbf{A}_{k l}^{-1} \mathbf{W}_{l} \mathbf{A}_{k l}^{-1} \mathbf{W}_{k} \mathbf{A}_{k l}^{-1} \mathbf{J}\right]\right)\right\} .
\end{aligned}
$$


${ }^{1}$ Yu. Ralchenko, A. E. Kramida, and J. Reader, NIST Atomic Spectra Database, version 3.1.5 (April 2008), URL http://physics.nist.gov/asd.

${ }^{2}$ Z.-C. Yan and G. W. F. Drake, Phys. Rev. A 52, 3711 (1995).

${ }^{3}$ P. J. Pelzl, G. J. Smethells, and F. W. King, Phys. Rev. E 65, 036707 (2002).

${ }^{4}$ D. M. Feldmann, P. J. Pelzl, and F. W. King, J. Math. Phys. 39, 6262 (1998).

${ }^{5}$ Z.-C. Yan, M. Tambasco, and G. W. F. Drake, Phys. Rev. A 57, 1652 (1998).

${ }^{6}$ Z.-C. Yan, W. Nörtershäuser, and G. W. F. Drake, Phys. Rev. Lett. 100 243002 (2008).

${ }^{7}$ M. Puchalski and K. Pachucki, Phys. Rev. A 73, 022503 (2006).

${ }^{8}$ M. Stanke, D. Kedziera, S. Bubin, and L. Adamowicz, Phys. Rev. Lett. 99, 043001 (2007).

${ }^{9}$ S. Bubin, J. Komasa, M. Stanke, and L. Adamowicz, Phys. Rev. A 81, 052504 (2010).
${ }^{10}$ S. Bubin, J. Komasa, M. Stanke, and L. Adamowicz, J. Chem. Phys. 132, 114109 (2010).

${ }^{11}$ S. Bubin, M. Stanke, and L. Adamowicz, J. Chem. Phys. 131, 044128 (2009).

${ }^{12}$ K. L. Sharkey, M. Pavanello, S. Bubin, and L. Adamowicz, Phys. Rev. A 80, 062510 (2009)

${ }^{13}$ K. L. Sharkey, S. Bubin, and L. Adamowicz, J. Chem. Phys. 132, 184106 (2010).

${ }^{14}$ S. Bubin, J. Komasa, M. Stanke, and L. Adamowicz, J. Chem. Phys. 131, $234112(2009)$

${ }^{15}$ M. Stanke, J. Komasa, S. Bubin, and L. Adamowicz, Phys. Rev. A 80, 022514 (2009).

${ }^{16}$ M. Stanke, J. Komasa, D. Kedziera, S. Bubin, and L. Adamowicz, Phys. Rev. A 77, 062509 (2008). 